\title{
Antitumor effect of FP3 in a patient-derived tumor tissue xenograft model of gastric carcinoma through an antiangiogenic mechanism
}

\author{
KETAO JIN ${ }^{1-3^{*}}$, HUANRONG LAN ${ }^{4 *}$, FEILIN CAO $^{1 *}$, ZHENZHEN XU $^{2}$, NA HAN $^{5}$, \\ GUANGLIANG $\mathrm{LI}^{2}$, KUIFENG $\mathrm{HE}^{2}$ and LISONG TENG ${ }^{2}$
}

\begin{abstract}
${ }^{1}$ Department of Surgical Oncology, Taizhou Hospital, Wenzhou Medical College, Linhai, Zhejiang 317000;
${ }^{2}$ Department of Surgical Oncology, First Affiliated Hospital, College of Medicine, Zhejiang University, Hangzhou, Zheijiang;

${ }^{3}$ Department of Surgery, Affiliated Zhuji Hospital, Wenzhou Medical College, Zhuji, Zhejiang; ${ }^{4}$ Department of Gynecology and Obstetrics, Taizhou Hospital, Wenzhou Medical College, Linhai, Zhejiang; ${ }^{5}$ Cancer Chemotherapy Center, Zhejiang Cancer Hospital, Zhejiang University of Chinese Medicine, Hangzhou, Zhejiang 310003, P.R. China
\end{abstract}

Received December 13, 2011; Accepted January 30, 2012

DOI: $10.3892 / \mathrm{ol} .2012 .603$

\begin{abstract}
FP3 (KH902/KH903) is a novel vascular endothelial growth factor (VEGF) blocker with antiangiogenic properties. Previous studies revealed that FP3, a humanized fusion protein that combines ligand binding elements from the extracellular domains of VEGF receptors 1 and 2 and the Fc portion of IgG1, has an inhibitory effect on the VEGFmediated proliferation and migration of human umbilical vein endothelial cells, and VEGF-mediated vessel sprouting of rat aortic rings in vitro. Thus, FP3 was considered as a new promising agent in treating human choroidal neovascularization (CNV) caused by age-related macular degeneration (AMD). FP3 also has an antitumor effect in a non-small cell lung cancer cell line (A549) xenograft model in nude mice. However, little is known of the direct effects of FP3 on tumor vessels. In this study, we investigated the effects of FP3 on blood vessels in a patient-derived tumor tissue (PDTT) xenograft model of gastric carcinoma, using large tumors with established vasculature. Treatment with FP3 caused robust and early changes in endothelial cells and pericytes of vessels in the PDTT xenograft model. Vascular density decreased and vascular sprouting was suppressed by treatment with FP3. Pericytes did not degenerate to the same extent as endothelial cells, and those on surviving tumor vessels achieved a more normal phenotype. Our results revealed that FP3 has a direct and rapid antiangiogenic effect on tumor vessels, which was
\end{abstract}

Correspondence to: Dr Lisong Teng, Department of Surgical Oncology, First Affiliated Hospital, College of Medicine, Zhejiang University, 79 Qingchun Road, Hangzhou, Zhejiang 310003, P.R. China

E-mail: jinketao2001@zju.edu.cn

*Contributed equally

Key words: antiangiogenic effect, antitumor effect, FP3, gastric carcinoma, vascular endothelial growth factor achieved mainly via regression of tumor vasculature, inhibition of new and recurrent vessel growth, and normalization of existing tumor vasculature.

\section{Introduction}

Angiogenesis is one of the eight hallmarks of cancer which are gained during the multistep development of human tumors (1). Similar to normal tissues, tumors require sustenance in the form of nutrients and oxygen as well as an ability to evacuate metabolic wastes and carbon dioxide. The tumor-associated neovasculature, generated by the process of angiogenesis, addresses these requirements. Tumors are known to achieve vasculature by endothelial cell sprouting (2), cooption of preexisting vessels (3-6), intussusceptive microvascular growth $(7,8)$, postnatal vasculogenesis (9), glomeruloid angiogenesis $(10)$ or vasculogenic mimicry $(11,12)$. Vascular endothelial growth factor (VEGF) is well-established as a key mediator in some or all of these processes. Furthermore, VEGF is the only angiogenic factor known to be present throughout the entire tumor lifecycle $(13,14)$. Based on this evidence, VEGF is considered as a rational target for antiangiogenic drug development (14-16). Since anti-VEGF approaches act by blocking tumor-associated angiogenesis, which appears to be widely required by a number of different types of tumor, these approaches have been shown to be useful against a wide variety of solid tumors $(16,17)$.

FP3 (KH902/KH903) is an engineered protein which contains the extracellular domain 2 of VEGF receptor 1 (Flt-1) and the extracellular domains 3 and 4 of VEGF receptor 2 (Flk-1, KDR), fused to the Fc portion of human immunoglobulin G1 $(16,18)$. Recent studies have indicated that FP3 showed promise as a local antiangiogenic treatment of human choroidal neovascularization (CNV) caused by age-related macular degeneration (AMD) $(16,19-21)$. In subsequent studies, it was demonstrated that FP3 has an inhibitory effect on VEGF-mediated proliferation and migration of human umbilical vein endothelial cells, and on the VEGF-mediated 
vessel sprouting of rat aortic rings in vitro (18). FP3 also exhibits an antitumor effect in a non-small cell lung cancer cell line (A549) xenograft model in nude mice (18). However, little is known of the effects of FP3 on tumor vessels.

Measurement of microvascular density is one of the most common microscopic methods used to quantify angiogenesis in tumors, as performed in a previous study (18). However, it is not always an accurate measure of efficacy as tumor mass may decrease in parallel with the number of blood vessels (22). Tumor burden, another standard endpoint, provides limited insight into whether drugs act on blood vessels or tumor cells, and may not reveal whether tumor growth is stabilized by angiogenesis inhibition. Thus, new methods are required for evaluating the vascular effects of FP3.

In the present study, we examined the cellular effects of FP3 on blood vessels, mainly focusing on the endothelial cells and pericytes of tumor vessels in a patient-derived tumor tissue (PDTT) xenograft model of gastric carcinoma, using large tumors with established vasculature. A fluorescent microscopic approach was applied to reveal the cellular morphologic changes of endothelial cells and pericytes of tumor vessels following administration with FP3.

\section{Materials and methods}

Patients and tissue samples. Tumor samples were obtained at initial surgery from a 57-year-old male patient with gastric carcinoma. Prior written informed consent was obtained from the patient and the study received ethics board approval from the First Affiliated Hospital, College of Medicine, Zhejiang University, China. The patient had not received chemotherapy or radiation therapy prior to surgery. The histological type was determined according to WHO criteria. The metastatic tumor was diagnosed as diffuse infiltrative signet ring cell carcinoma. The tumor sample was placed into RPMI-1640 medium immediately following surgical resection under sterile conditions, and transported without delay to the animal facility.

Reagents and drugs. The antibody against platelet endothelial cell adhesion molecule-1 (PECAM-1, CD31; rat monoclonal, clone MEC 13.3) was purchased from BD Pharmingen (San Diego, CA, USA). The antibody against $\alpha$-smooth muscle actin ( $\alpha$-SMA, rabbit polyclonal) was purchased from Abcam (Cambridge, UK). The fluorescent (Cy3- or FITC-conjugated) secondary antibodies (goat anti-rat or goat anti-rabbit) were purchased from Jackson ImmunoResearch (West Grove, PA, USA). The RPMI-1640 medium, fetal bovine serum (FBS), penicillin and streptomycin were purchased from Gibco (Grand Island, NY, USA). Isofluorane, diethyl ether, ketamine, xylazine, paraformaldehyde and bovine serum albumin (BSA) were purchased from Sigma (St. Louis, MO, USA). Bevacizumab (Avastin) was purchased from Roche, Inc. (Roche, South San Francisco, CA, USA). FP3 was kindly provided as a gift from Kanghong Biotechnology Inc. (Konghong, Chengdu, China).

Establishment of PDTT xenograft model of gastric carcinoma. Four- to six-week-old female BALB/c nude mice were purchased from Slaccas (Shanghai Laboratory Animal Center, Shanghai, China). The mice were housed in a barrier facility and acclimatized to 12-h light-dark cycles for at least three days prior to use. The use of experimental animals adhered to the 'Principles of Laboratory Animal Care' (NIH publication no. 85-23, revised in 1985). Experiments were approved by the Institutional Animal Care and Use Committee of Zhejiang University [approval ID: SYXK(ZHE)2005-0072]. A PDTT xenograft model of gastric carcinoma was established for this study, as described in a previous study (23-26).

Treatments. Xenografts from the second mouse-to-mouse passage were allowed to grow to a size of $100-150 \mathrm{~mm}^{3}$, at which time (10 days after xenograft) mice were randomized into 3 cohorts. In cohort 1, 30 mice were divided into 3 groups of 10 mice; i) control (100 $\mu \mathrm{l}$ saline, intravenously (i.v.), twice per week); ii) bevacizumab (10 mg/kg, i.v., twice per week); iii) FP3 (15 mg/kg, i.v., twice per week). Mice were treated during the following 25 days. Tumor size was evaluated every four days by caliper measurements using the formula: tumor volume $=\left(\right.$ length $\mathrm{x}$ width $\left.{ }^{2}\right) / 2$. Experiments were terminated on day 34 . In cohort 2, 4 groups of female athymic nude mice $(n=40)$ were implanted with tumors for a further 3 weeks. One group $(n=10)$ was sacrificed for evaluation without treatment, the second group $(n=10)$ was treated with FP3 $(15 \mathrm{mg} / \mathrm{kg}$ body weight) twice per week for 3 weeks, and the third group $(n=10)$ was treated with FP3 (15 mg/kg body weight) twice per week for 3 weeks. The treatment was stopped for a 2 week period of recovery (total 5 weeks before experiments were terminated). The fourth group $(\mathrm{n}=10)$ was treated with FP3 $(15 \mathrm{mg} / \mathrm{kg}$ body weight) twice per week for 3 weeks, then stopped for a recovery of 2 weeks followed by 2 weeks of further treatment ( 3 and 2 weeks of treatment, total 7 weeks of experiment duration). In cohort 3, 3 groups of female athymic nude mice ( $n=30,10$ mice per group) were implanted with tumors for a further 3 weeks. One group (day 0) was sacrificed for vascular morphology evaluation without treatment, whereas each of the other 2 groups was treated with a single dose of FP3 $(15 \mathrm{mg} / \mathrm{kg})$, and sacrificed for evaluation on the third day (day 2) and the fifth day (day 4) following treatment.

Fixation by vascular perfusion. Selected mice were anesthetized with ketamine $(87 \mathrm{mg} / \mathrm{kg}$ ) plus xylazine $(13 \mathrm{mg} / \mathrm{kg})$, which was injected intramuscularly. The chest was rapidly opened, and the vasculature was perfused for $3 \mathrm{~min}$ at a pressure of $120 \mathrm{mmHg}$ with fixative [4\% paraformaldehyde in $0.1 \mathrm{~mol} / \mathrm{l}$ phosphate-buffered saline (PBS), $\mathrm{pH}$ 7.4] from an 18-gauge cannula, which was inserted into the aorta via an incision in the left ventricle. Blood and fixative exited through an opening in the right atrium. Following perfusion, the implanted tumor was removed and placed into fixative for $2 \mathrm{~h}$ at $4^{\circ} \mathrm{C}$. Samples were then rinsed several times with PBS, infiltrated overnight with $30 \%$ sucrose, embedded in optimal cutting temperature (OCT) medium, and frozen for cryostat sectioning (27).

Immunohistochemistry. Cryostat sections (8 to $10 \mu \mathrm{m}$ ) were brought to room temperature, air dried overnight and fixed in acetone for $10 \mathrm{~min}$. Slides were allowed to air dry for $30 \mathrm{~min}$, and were washed 3 times for $5 \mathrm{~min}$ each in PBS. Samples were then incubated in 5\% BSA in PBS for $30 \mathrm{~min}$ at room temperature to block non-specific antibody binding. The sections were then incubated overnight at room temperature in humidified chambers in combinations 
of two primary antibodies (CD31, 1:40; and $\alpha$-SMA, 1:200) diluted in PBS. After several rinses with PBS, specimens were incubated for $1 \mathrm{~h}$ at room temperature with fluorescent (Cy3- or FITC-conjugated) secondary antibodies (goat antirat or goat anti-rabbit) diluted (1:200) in PBS. Specimens were rinsed again with PBS, and mounted in Vectashield mounting medium (Vector Laboratories, Burlingame, CA, USA) $(28,29)$. Tissue sections were then examined and images were digitally captured using a Zeiss Axiophot fluorescence microscope (Carl Zeiss, Thornwood, NY, USA), equipped with single, dual and triple fluorescence filters and a lowlight, externally cooled, three-chip charge-coupled device (CCD) camera (480x640 pixel RGB-color images, CoolCam; SciMeasure Analytical Systems, Atlanta, GA, USA), and saved as TIFF files.

Statistical analysis. Data were presented as the mean \pm SEM and analyzed by SPSS 16.0 software. Differences among the means of the groups were determined using one-way ANOVA tests. $\mathrm{P}<0.05$ is considered to indicate a statistically significant difference.

\section{Results}

FP3 markedly blocks gastric carcinoma growth in vivo. We examined the effect of FP3 on the growth of the established PDTT xenograft model of gastric carcinoma. Following implantation, tumors were allowed to grow for 10 days, forming large retroperitoneal tumors of $>100 \mathrm{~mm}^{3}$. Injections of FP3 (15 mg/kg body weight), Avastin (10 mg/kg body weight) or saline were then administered i.v. twice per week for 25 days, after which the animals were sacrificed, and the tumors were excised and measured. FP3 significantly inhibited the growth of tumors (Fig. 1). The effects of Avastin were evaluated for comparison.

FP3 results in regression of tumor vasculature. To evaluate the effects of FP3 on tumor-associated angiogenesis, the tumors from the abovementioned study on cohort 1 mice were sectioned and immunostained with antibodies to CD31 and $\alpha$-SMA, in order that the vasculature could be visualized (Fig. 2). This analysis revealed that vasculature was almost absent in FP3-treated xenografts. FP3 (treatment for 25 days) almost completely blocked tumorassociated angiogenesis, with the stunted tumors being largely avascular (Fig. 2B,E and H). In contrast to the FP3-treated tumors, control tumors in saline-treated mice were not only much larger, but also had a high vascular density (Fig. 2A,D and G). These results indicated that FP3 administration reduces xenograft size and concurrently causes decreased microvessel sprouting.

FP3 inhibits new and recurrent vessel growth. Inhibition of VEGF signaling by FP3 blocks angiogenesis and reduces tumor vascularity. However, little is known regarding the events after treatment ends. We subsequently evaluated the effects of FP3 on the growth of new and recurrent tumor vessels (Fig. 3). Blood vessels in untreated tumors were abundant, tortuous and variable in diameter (Fig. 3A,E and I). The vascularity of the tumors was conspicuously reduced following

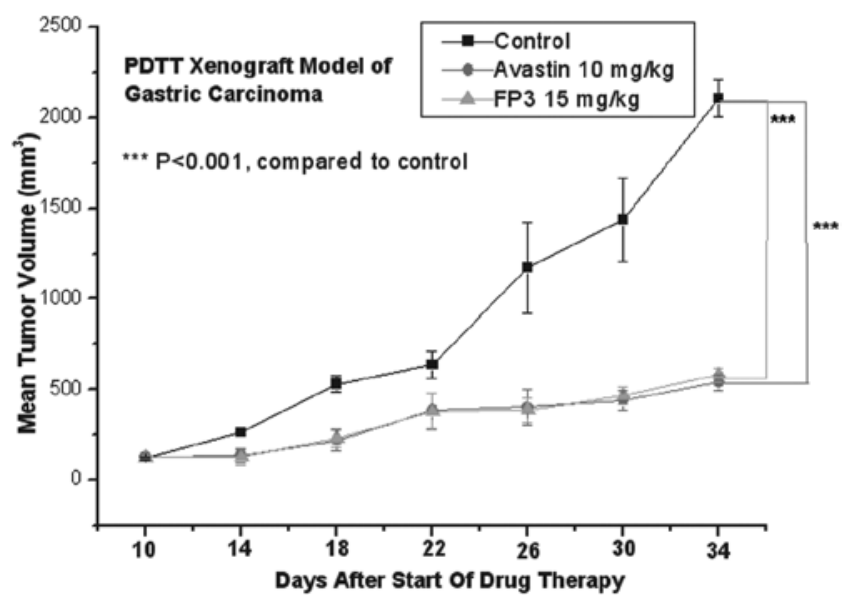

Figure 1. Response curve of FP3 and Avastin in the PDTT xenograft model of gastric carcinoma. A total of 10 mice per group were treated with the corresponding agent according to the Materials and methods. Data shown are the means \pm SEM. The differences between the control tumor volumes, FP3and Avastin-treated tumor volumes were analyzed using one-way ANOVA tests. ${ }^{* * *} \mathrm{P}<0.001$, versus control. Experiments were repeated at least twice with similar results. PDTT, patient-derived tumor tissue.

treatment with FP3 for 3 weeks (Fig. 3B,F and J), but were as abundant as before the treatment after withdrawal of FP3 for 2 weeks (Fig. 3C,G and K). However, after treatment with FP3 for another 2 weeks $(3+2$ weeks, treatment lasted 3 weeks, then stopped for a recovery of 2 weeks followed by 2 weeks of further treatment), tumor vessels were less tortuous, more uniform in caliber, and had fewer branches and sprouts (Fig. 3D,H and L).

Following treatments with FP3 for 3 weeks and 5 weeks $(3+2$ weeks, treatment lasted 3 weeks, then stopped for a recovery of 2 weeks followed 2 weeks of treatment further), the majority of surviving vessels in tumors did not have sprouts (Fig. 3B,D,F,H,J and L). Endothelial sprouts, appearing as cell protrusions tipped by filopodia, were abundant on blood vessels in untreated tumors as well as in tumors treated with FP3 for 3 weeks and stopped for a recovery of 2 weeks (Fig. 3A,C,E,G,I and K). These results showed that FP3 has a marked antiangiogenic effect on preexisting or newly formed vessels.

FP3 normalizes existing tumor vasculature. To determine whether the abovementioned results were a direct effect of FP3 on tumor vessels, we examined its effect in a short-term assay using large tumors with established vasculature. The effect of VEGF inhibition on microvessel density, pericyte coverage and tumor vessel phenotype on the third day (day 2) and fifth days (day 4) following the administration of a single dose of FP3 $(15 \mathrm{mg} / \mathrm{kg})$ to mice with established PDTT xenograft was examined. Tumors from selected mice, which were sacrificed at each of these times, demonstrated a significant, rapid and progressive decrease in tumor microvessel density, as assessed by CD31 immunohistochemistry (Fig. 4A-C).

Serial tumor sections were also stained for $\alpha$-SMA to identify perivascular smooth muscle cells. Unlike the marked loss of endothelial cells in treated tumors, the number of pericytes remained stable after FP3 treatment (Fig. 4E and F). This observation suggests that there was a selective loss of 
NS

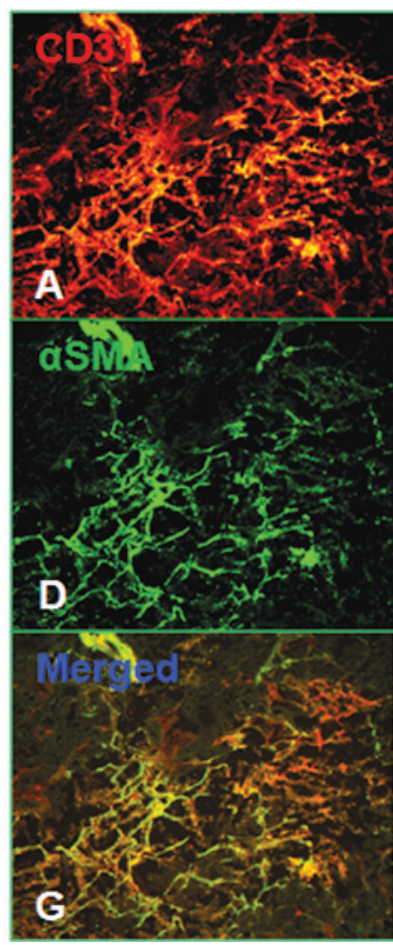

FP3 $15 \mathrm{mg} / \mathrm{kg} \quad$ Avastin $10 \mathrm{mg} / \mathrm{kg}$

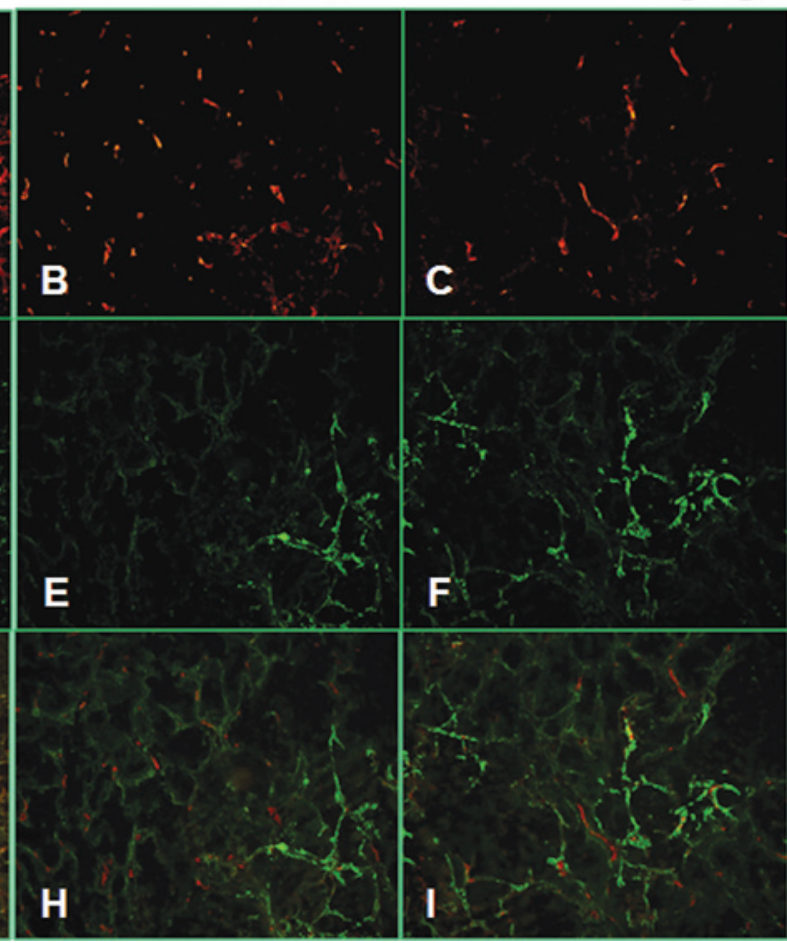

Figure 2. FP3 decreased the vascular structure. The vasculature was examined by angiography with immunostaining for endothelial cells (using anti-CD31 antibody; bar, $100 \mu \mathrm{m}$ ), and pericytes (using anti- $\alpha$-SMA antibody; bar, $100 \mu \mathrm{m}$ ). (A, D and G) The control tumors in saline-treated mice were larger and exhibited high vascular density. (B, E and H) FP3 treatment blocked tumor-associated angiogenesis, with the stunted tumors being largely avascular (C, F and I) Bevacizumab (Avastin) treatment blocked tumor-associated angiogenesis. NS, normal saline.

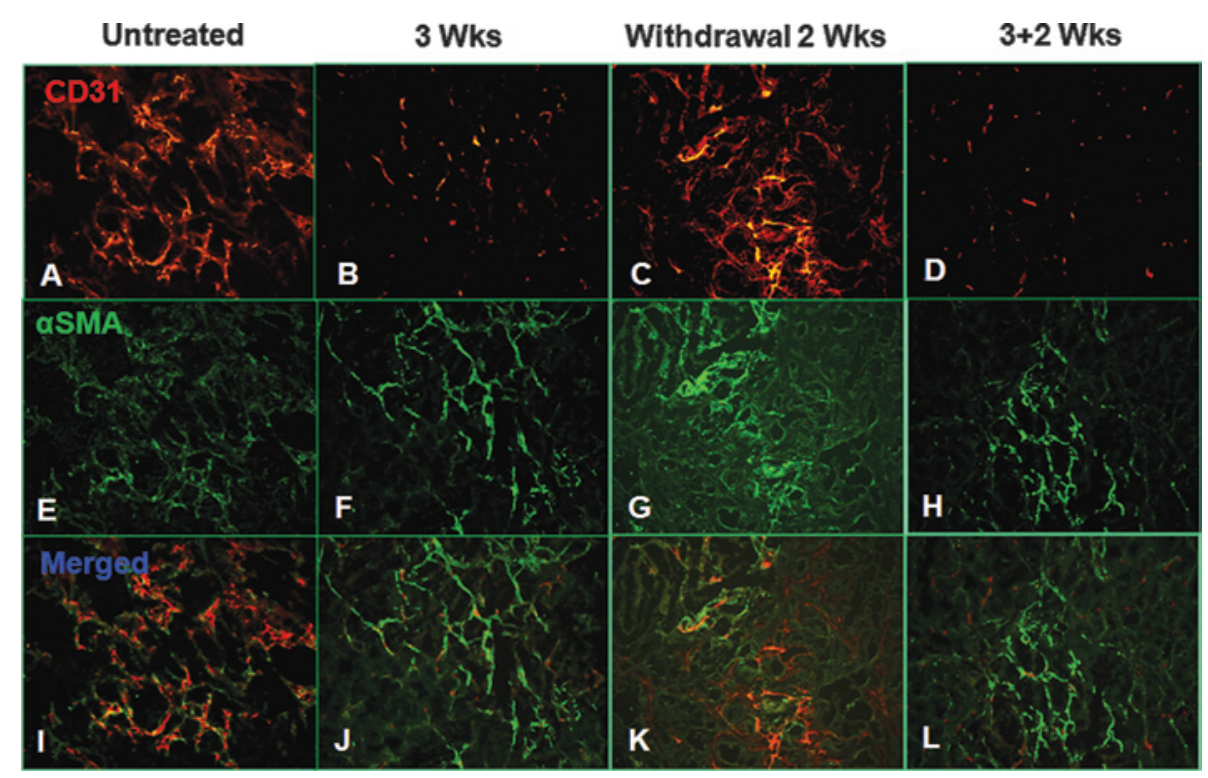

Figure 3. Effect of the different durations of FP3 treatment on the morphology of the tumor vessels in established PDTT xenograft of gastric carcinoma. Representative fluorescent microscopic images (bar, $100 \mu \mathrm{m}$ ) show: (A, E and I) vasculature in mice prior to FP3 administration; (B, F and J) following FP3 administration for 3 weeks; (C, G and K) following FP3 administration for 3 weeks and stopped for a recovery of 2 weeks; and (D, H and L) following FP3 administration for $3+2$ weeks (see Materials and methods). PDTT, patient-derived tumor tissue.

the more immature vessels that lacked pericytes, whereas the more mature vessels remained. One population of pericytes became closely associated with surviving vessels (Fig. 5). These pericytes were more tightly apposed to the endothelial cells compared to the untreated tumors (Fig. 5). Unlike the majority of pericytes in untreated tumors, some were oriented circumferentially around the vessels (Fig. 5B,D and F), resembling smooth muscle cells on arterioles. Another population of $\alpha$-SMA-positive cells had no apparent association with blood vessels. A number of of these cells did not colocalize with 
Day 0
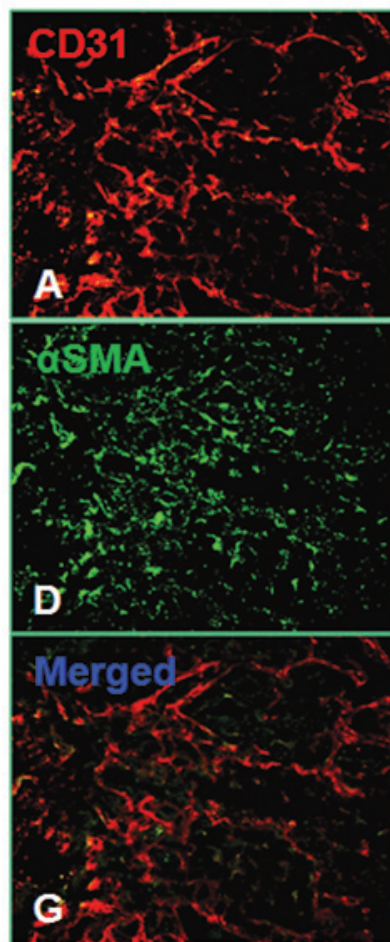

Day 2
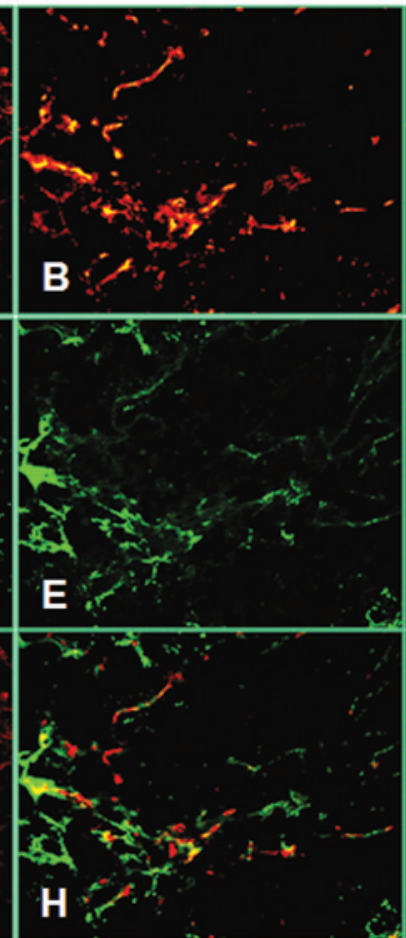

Day 4
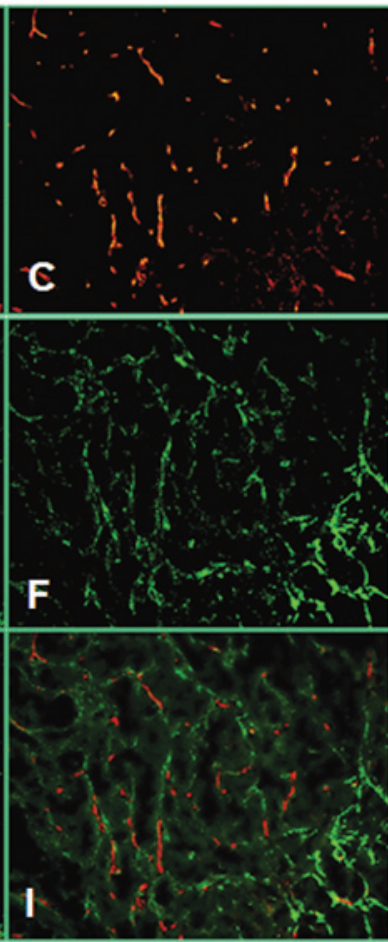

Figure 4. Effect of a single dose of FP3 on the morphology of the tumor vessels in established PDTT xenograft of gastric carcinoma. Representative fluorescent microscopic images (bar, $100 \mu \mathrm{m}$ ) show: (day 0; A, D and G) vasculature in mice prior to administration of FP3 (15 mg/kg); and (day 2; B, E and H) 3 days and (day 4; C, F and I) 5 days following administration of FP3 $(15 \mathrm{mg} / \mathrm{kg})$. PDTT, patient-derived tumor tissue.

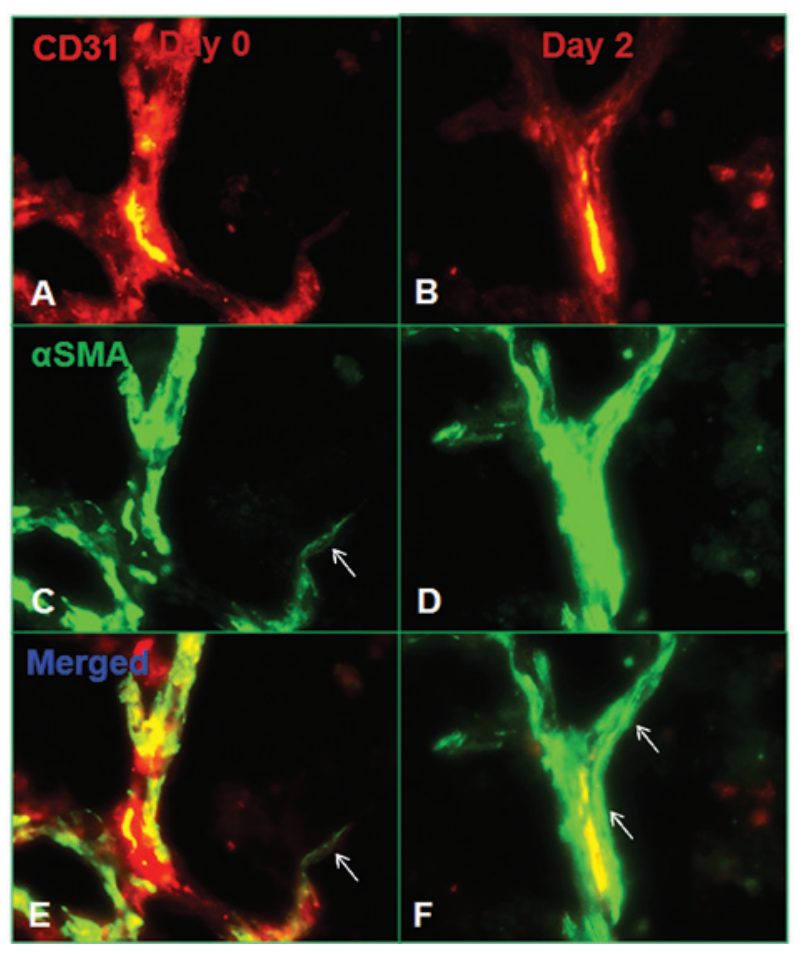

Figure 5. Tightening of pericytes on tumor vessels following the inhibition of VEGF signaling by FP3. Representative fluorescent microscopic images (bar, $100 \mu \mathrm{m}$ ) show changes in pericyte-endothelial cell relationships (arrows) in tumor vessels. (A, C and E) In tumor vessels, $\alpha$-SMA immunoreactive pericytes (green) are loosely attached to CD31-positive endothelial cells (red). (B, D and F) By comparison, following treatment with FP3 $(15 \mathrm{mg} / \mathrm{kg})$ for 2 days, $\alpha$-SMA-positive pericytes are tighter on endothelial cells visualized by immunofluorescence. VEGF, vascular endothelial growth factor. the surviving CD31-positive blood vessels (Fig. 5A,C and E). As evidence of the greater impact of treatment on endothelial cells compared to pericytes, the reduction in CD31 immunoreactivity was greater than that in $\alpha$-SMA immunoreactivity. These results indicate that during the treatment period, tumor microvessel density decreased and pericyte coverage of vessels increased, confirming the hypothesis of vessel normalization. This 'normalized' vasculature is characterized by less leaky, less dilated and less tortuous vessels, with a greater coverage by pericytes.

\section{Discussion}

FP3 is a humanized fusion protein that combines ligand binding elements from the extracellular domains of VEGF receptors 1 and 2 and the $\mathrm{Fc}$ portion of $\mathrm{IgG1}$, and is able to bind to all types of VEGF-A $(16,18)$. A previous study demonstrated that FP3 significantly blocked the growth of a non-small cell lung cancer cell line (A549) tumor in a subcutaneous xenograft model, and markedly decreased the vessel density of the tumor (18). These results were further confirmed in the present study in a PDTT xenograft model of gastric carcinoma (Figs. 1 and 2). These preclinical observations have indicated that FP3 is capable of causing regression of existing tumor vasculature and significant reductions in tumor vascular volume and density.

Although existing vasculature is essential for tumor survival, tumors are unable to grow and spread without the generation of new vasculature (14). Our findings have shown that FP3 resulted in almost complete inhibition of new vessel 
growth, which is required for tumor growth and metastasis. We also found that withdrawal of FP3 resulted in the regrowth of tumor vessels. However, this regrowth was inhibited again by further treatment of FP3, indicating that FP3 may result in the ongoing inhibition of recurrent tumor vessel growth. In terms of clinical significance, inhibition of recurrent tumor vessel growth by FP3 may provide continued disease control.

The common hypothesis that antiangiogenic therapy eradicates tumor vasculature, thus depriving the tumor of oxygen and nutrients necessary for survival, has been challenged by certain studies. These studies suggest that treatment with antiangiogenic agents is able to transiently reverse some of the abnormalities of tumor vessels or 'normalize' the tumor vasculature for a short period of time, thereby providing a window of opportunity for improving drug delivery and enhancing sensitivity to conventional chemotherapy and radiation treatment $(17,22,30-32)$. Based on our previous results and those of the present study, it is hypothesized that FP3 also has the potential to 'normalize' the tumor vasculature, which is unique to this class of agents. To determine this event, we assessed the direct cell effects of FP3 on tumor vessels focusing on the endothelial cells and pericytes, by examining the effect of FP3 on a short-term assay using large tumors with established vasculature. A PDTT xenograft model of gastric carcinoma was used for this purpose. We focused on changes occurring during the first 5 days of treatment of established tumors to identify primary vascular effects of FP3. Using a fluorescent microscopic approach, we found that FP3 significantly altered tumor vasculature, causing a rapid, quantitatively significant decrease in the number of microvessels in as short a time as 2 days. However, pericytes did not degenerate to the same extent as endothelial cells, and those on surviving tumor vessels achieved an increasingly normal phenotype. Pericytes have multiple abnormalities, including a loose association with endothelial cells. Treatment with FP3 normalized the phenotype of specific pericytes, as manifested by a tighter association with endothelial cells of surviving vessels. Following treatment with FP3, fewer pericytes regressed compared to endothelial cells. Numerous surviving $\alpha$-SMA-positive cells were not associated with tumor vessels, presumably due to the fact that their endothelial cells had degenerated (Figs. 4 and 5). Our results indicate that blockage of VEGF signaling with FP3 passively prunes the immature and leaky growing blood vessels of transplanted tumors in mice, causing an increased proportion of mature, functional vessels, and actively remodeling the remaining vasculature to an increasingly normal state.

The unregulated nature of tumor angiogenesis leads to the production of structurally and functionally abnormal vasculature, characterised by a number of different features, including increased vessel density, diameter, length and tortuosity, abnormally high interstitial fluid pressure, and increased vascular permeability $(33,34)$. These abnormalities prevent the effective delivery of therapy to the tumor. For example, the entry of large molecules, including chemotherapeutic agents, into the tumor would be impeded and hypoxia results from an inconsistent oxygen supply within the tumor, producing regions that would be resistant to radiotherapy and certain cytotoxic agents. The abovementioned vessel normalization effect of FP3 may help make tumor cells increasingly sensitive to cytotoxic chemotherapy and maximize the effectiveness of the overall cancer treatment strategy. In terms of clinical significance, normalization of tumor vasculature by FP3 may also maximize the efficacy of concomitant therapy.

In conclusion, this study demonstrated that FP3 has a direct and rapid antiangiogenic effect in solid tumors. The antiangiogenic effects of FP3 were demonstrated via the regression of tumor vasculature, inhibition of new and recurrent vessel growth, and normalization of existing tumor vasculature. Whether these morphological changes may be accompanied by functional changes (including decreased interstitial fluid pressure, increased tumor oxygenation, and improved penetration of drugs in solid tumors), and functional consequences with regard to the intratumoral delivery and antitumor activity of adjuvant anticancer agents when administered together with FP3, are unknown and should be ascertained in future studies.

\section{Acknowledgements}

This study was supported by the State Key Basic Research and Development Program of China (973 Program, Grant No. 2009CB521704), the National High-Tech Research and Development Program of China (863 Program, Grant No. 2006AA02A245), the National Natural Science Foundation of China (Grant No. 81000894), the Zhejiang Provincial Science and Technology Project (Grants No. 2009C13021, 2011C23087), the Science Research Fund of Shaoxing (Grants No. 2011D10013) and the Science Research Fund of Zhuji (Grants No. 2011CC7874). The funding groups had no role in the study design, data collection and analysis, decision to publish, or preparation of the manuscript.

\section{References}

1. Hanahan D and Weinberg RA: Hallmarks of cancer: the next generation. Cell 144: 646-674, 2011.

2. Paku S and Paweletz N: First steps of tumor-related angiogenesis. Lab Invest 65: 334-346, 1991.

3. Holash J, Maisonpierre PC, Compton D, Boland P, Alexander CR, Zagzag D, Yancopoulos GD and Wiegand SJ: Vessel cooption, regression, and growth in tumors mediated by angiopoietins and VEGF. Science 284: 1994-1998, 1999.

4. Döme B, Paku S, Somlai B and Tímár J: Vascularization of cutaneous melanoma involves vessel co-option and has clinical significance. J Pathol 197: 355-362, 2002.

5. Vermeulen PB, Colpaert C, Salgado R, Royers R, Hellemans H, Van Den Heuvel E, Goovaerts G, Dirix LY and Van Marck E: Liver metastases from colorectal adenocarcinomas grow in three patterns with different angiogenesis and desmoplasia. J Pathol 195: 336-342, 2001.

6. Paku S, Kopper L and Nagy P: Development of the vasculature in 'pushing-type' liver metastases of an experimental colorectal cancer. Int J Cancer 115: 893-902, 2005.

7. Kurz H, Burri PH and Djonov VG: Angiogenesis and vascular remodeling by intussusception: from form to function. News Physiol Sci 18: 65-70, 2003.

8. Osawa M, Masuda M, Kusano K and Fujiwara K: Evidence for a role of platelet endothelial cell adhesion molecule-1 in endothelial cell mechanosignal transduction: is it a mechanoresponsive molecule? J Cell Biol 158: 773-785, 2002.

9. Sundberg C, Nagy JA, Brown LF, Feng D, Eckelhoefer IA, Manseau EJ, Dvorak AM and Dvorak HF: Glomeruloid microvascular proliferation follows adenoviral vascular permeability factor/vascular endothelial growth factor-164 gene delivery. Am J Pathol 158: 1145-1160, 2001.

10. Asahara T and Kawamoto A: Endothelial progenitor cells for postnatal vasculogenesis. Am J Physiol Cell Physiol 287: $572-579,2004$ 
11. Maniotis AJ, Folberg R, Hess A, Seftor EA, Gardner LM Pe'er J, Trent JM, Meltzer PS and Hendrix MJ: Vascular channel formation by human melanoma cells in vivo and in vitro: vasculogenic mimicry. Am J Pathol 155: 739-752, 1999.

12. Hendrix MJ, Seftor EA, Hess AR and Seftor RE: Vasculogenic mimicry and tumour-cell plasticity: lessons from melanoma. Nat Rev Cancer 3: 411-421, 2003.

13. Ferrara N: Vascular endothelial growth factor: basic science and clinical progress. Endocr Rev 25: 581-611, 2004.

14. Hicklin DJ and Ellis LM: Role of the vascular endothelial growth factor pathway in tumor growth and angiogenesis. J Clin Oncol 23: 1011-1027, 2005.

15. Ferrara N, Gerber HP and LeCouter J: The biology of VEGF and its receptors. Nat Med 9: 669-676, 2003.

16. Teng LS, Jin KT, He KF, Wang HH, Cao J and Yu DC: Advances in combination of antiangiogenic agents targeting VEGF-binding and conventional chemotherapy and radiation for cancer treatment. J Chin Med Assoc 73: 281-288, 2010.

17. Teng LS, Jin KT, He KF, Zhang J, Wang HH and Cao J: Clinical applications of VEGF-trap (aflibercept) in cancer treatment. J Chin Med Assoc 73: 449-456, 2010.

18. Jin K, He K, Teng F, Li G, Wang H, Han N, Xu Z, Cao J, Wu J, Yu D and Teng L: FP3: a novel VEGF blocker with antiangiogenic effects in vitro and antitumour effects in vivo. Clin Transl Oncol 13: 878-884, 2011.

19. Zhang M, Zhang J, Yan M, Li H, Yang C and Yu D: Recombinant anti-vascular endothelial growth factor fusion protein efficiently suppresses choridal neovasularization in monkeys. Mol Vis 14 37-49, 2008

20. Zhang M, Yu D, Yang C, Xia Q, Li W, Liu B and Li H: The pharmacology study of a new recombinant human VEGF receptor-fc fusion protein on experimental choroidal neovascularization. Pharm Res 26: 204-210, 2009.

21. Zhang M, Zhang J, Yan M, Luo D, Zhu W, Kaiser PK, Yu DC and KH902 Phase 1 Study Group: A phase 1 study of KH902, a vascular endothelial growth factor receptor decoy, for exudative age-related macular degeneration. Ophthalmology 118: 672-678, 2011.

22. Inai T, Mancuso M, Hashizume H, Baffert F, Haskell A, Baluk P, Hu-Lowe DD, Shalinsky DR, Thurston G, Yancopoulos GD and McDonald DM: Inhibition of vascular endothelial growth factor (VEGF) signaling in cancer causes loss of endothelial fenestrations, regression of tumor vessels, and appearance of basement membrane ghosts. Am J Pathol 165: 35-52, 2004.
23. Jin K, Teng L, Shen Y, He K, Xu Z and Li G: Patient-derived human tumour tissue xenografts in immunodeficient mice: a systematic review. Clin Transl Oncol 12: 473-480, 2010.

24. Jin K, He K, Li G and Teng L: Personalized cancer therapy using a patient-derived tumor tissue xenograft model: a translational field worthy of exploring further? Pers Med 7: 597-606, 2010.

25. Jin K, He K, Teng F, Han N, Li G, Xu Z and Teng L: Heterogeneity in primary tumors and corresponding metastases: could it provide us with any hints to personalize cancer therapy? Pers Med 8: 175-182, 2011.

26. Jin K, He K, Han N, Li G, Wang H, Xu Z, Jiang H, Zhang J and Teng L: Establishment of a PDTT xenograft model of gastric carcinoma and its application in personalized therapeutic regimen selection. Hepatogastroenterology 58: 1814-1822, 2011.

27. Mancuso MR, Davis R, Norberg SM, O'Brien S, Sennino B, Nakahara T, Yao VJ, Inai T, Brooks P, Freimark B, Shalinsky DR, Hu-Lowe DD and McDonald DM: Rapid vascular regrowth in tumors after reversal of VEGF inhibition. J Clin Invest 116: 26102621,2006

28. Morikawa S, Baluk P, Kaidoh T, Haskell A, Jain RK and McDonald DM: Abnormalities in pericytes on blood vessels and endothelial sprouts in tumors. Am J Pathol 160: 985-1000, 2002.

29. Baluk P, Morikawa S, Haskell A, Mancuso M and McDonald DM: Abnormalities of basement membrane on blood vessels and endothelial sprouts in tumors. Am J Pathol 163: 1801-1815, 2003.

30. Willett CG, Boucher Y, Di Tomaso E, Duda DG, Munn LL, Tong RT, Chung DC, Sahani DV, Kalva SP, Kozin SV, et al: Direct evidence that the VEGF-specific antibody bevacizumab has antivascular effects in human rectal cancer. Nat Med 10: 145-147, 2004.

31. Baffert F, Le T, Sennino B, Thurston G, Kuo CJ, Hu-Lowe D and McDonald DM: Cellular changes in normal blood capillaries undergoing regression after inhibition of VEGF signaling. Am J Physiol Heart Circ Physiol 290: H547-559, 2006.

32. Byrne AT, Ross L, Holash J, Nakanishi M, Hu L, Hofmann JI, Yancopoulos GD and Jaffe RB: Vascular endothelial growth factor-trap decreases tumor burden, inhibits ascites, and causes dramatic vascular remodeling in an ovarian cancer model. Clin Cancer Res 9: 5721-5728, 2003

33. Jain RK: Normalizing tumor vasculature with anti-angiogenic therapy: a new paradigm for combination therapy. Nat Med 7: 987-989, 2001.

34. Jain RK: Normalization of tumor vasculature: an emerging concept in antiangiogenic therapy. Science 307: 58-62, 2005. 